\title{
Characterization of a Waveguide ECR Plasma Source
}

\author{
Brandon R. Weatherford ${ }^{1}$ and John E. Foster ${ }^{2}$ \\ University of Michigan, Ann Arbor,MI,48109
}

The design and preliminary testing of a waveguide electron cyclotron resonance (ECR) heated plasma source is presented in this paper. The design utilizes resonant absorption of traveling microwaves to heat the discharge, avoiding any lifetime constraints on the device due to barium depletion or ion bombardment in more conventional devices. Results from magnetostatic calculations are presented, which are used to design the magnetic field geometry with samarium cobalt permanent magnets. The results from microwave solver simulations used to finalize the waveguide design are presented. The source, which operates in the circular TE11 mode, produces a discharge through either ECR or upper hybrid heating, depending on the magnet geometry. Preliminary tests show that the self-starting source is capable of delivering $226 \mathrm{~mA}$ of current at an absorbed power level of $80 \mathrm{~W}$ and argon flow rate of $2.6 \mathrm{sccm}$. The strong magnetic field perpendicular to the desired direction of plasma flow confines the plasma quite well. However, it appears to be the limiting factor on the amount of extractable current along with the plasma density at the extraction electrode. Langmuir probe traces at the location of the extraction electrode suggest that the plasma density is highly peaked at low flow rates, while the electron temperature is nearly independent of flow rate over the gas flow range investigated.

\section{Nomenclature}

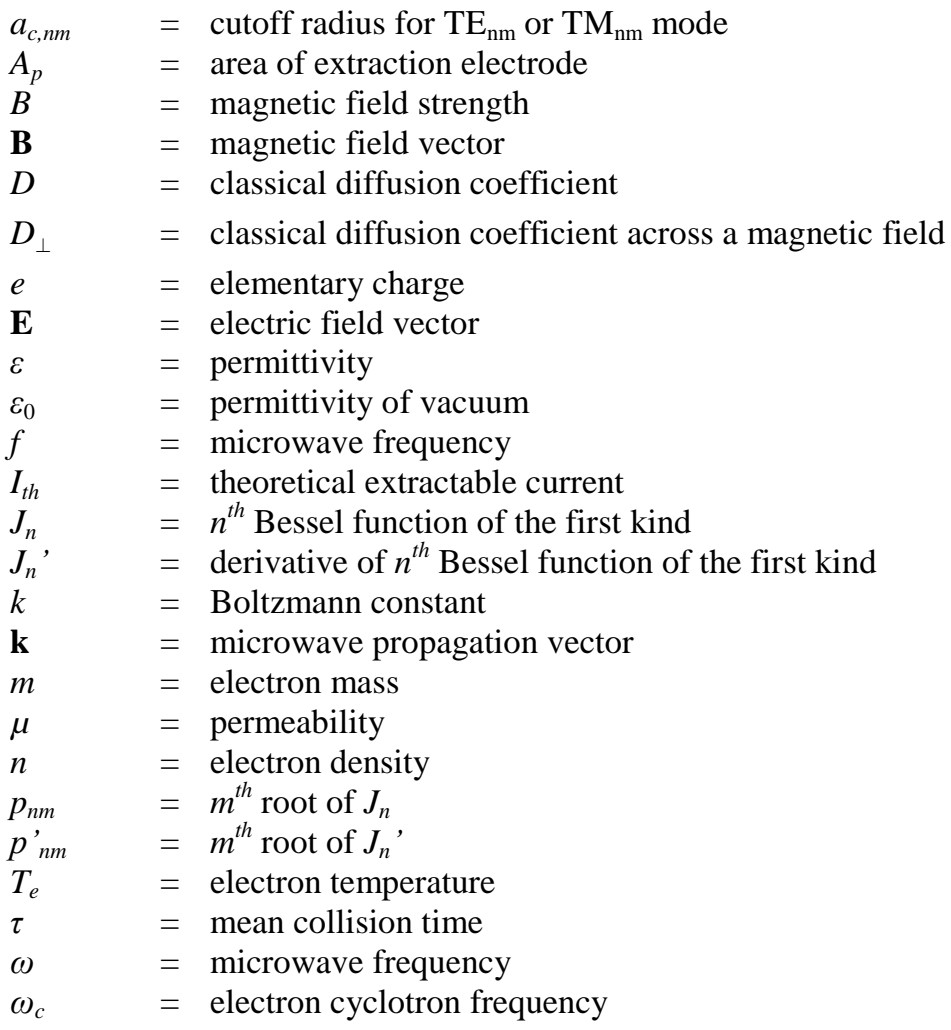

\footnotetext{
${ }^{1}$ Graduate Student, Student Member AIAA.

${ }^{2}$ Associate Professor, Senior Member AIAA.
} 
$\omega_{p e} \quad=$ electron plasma frequency

$\omega_{U H} \quad=$ upper hybrid frequency

\section{Introduction}

$\mathrm{E}^{\mathrm{s}}$ LECTRIC propulsion (EP) systems, like electrostatic gridded ion thrusters and Hall thrusters, are now wellestablished technologies which are often used for satellite station-keeping and, less frequently, exploratory missions that require a large specific impulse. The Deep Space 1, Hayabusa, SMART-1, and Dawn missions have all demonstrated the successful use of various EP systems in space flight. ${ }^{1-4}$ It is reasonable to expect that EP will be used as the primary propulsion method for future missions as well, particularly those to the outer planets and beyond.

In most of the recent ion and Hall thruster systems to date, the main discharge as well as the ion beam neutralizer have used a hollow cathode assembly (HCA) as the source of electrons. One major failure mechanism of the HCA is the depletion of emitter impregnate material due to evaporation and ion bombardment. Such depletion limits the usable lifetime of the HCA. It would therefore be useful to develop an electron source that does not employ thermionic emitters.

A microwave plasma discharge could be used as an alternative to the thermionic emitter for not only neutralizer applications but also for main discharge cathode duty. The Hayabusa mission successfully demonstrated the use of a microwave plasma for main discharge and neutralizer in space. The $\mu 10$ neutralizers used on Hayabusa employ a wire antenna that is in direct contact with the neutralizer source plasma. ${ }^{5}$ Although this design eliminates any barium depletion problems associated with an emissive insert, the antenna is still subject to ion bombardment.

Several varieties of microwave discharge plasma cathodes have been developed, including one that generated a discharge within an open-ended coaxial transmission line $e^{6}$, another that used the microwave electric field within a rectangular waveguide to produce the discharge ${ }^{7}$, and yet another that produced the discharge within a resonant cavity with an orifice ${ }^{8}$. Another set of approaches used permanent magnets to set up electron cyclotron resonance (ECR) heating zones, and used microwaves to heat the plasma discharge within these zones. In this vein, sources have been developed that utilize a coax-fed antenna ${ }^{9}$ or a magnetic grill assembly ${ }^{10}$ to create an ECR discharge. Also, a wire antenna has been used to generate an "off-resonance" discharge, in which the applied magnetic field is slightly stronger than that required for ECR breakdown. ${ }^{11}$ Substantial electron currents have been extracted from a high power neutralizer which employs electromagnets to set up ECR heating zones within a section of waveguide closed off by a cover plate with an extraction orifice. ${ }^{12}$

However, there has not yet been a microwave neutralizer source operating at low power that simultaneously can deliver the amount of electron current necessary for beam neutralization while avoiding the problem of lifetime limitations on the source due to erosion from ion bombardment. The purpose of this paper is to present the preliminary characterization of a $2.45 \mathrm{GHz}$ microwave plasma source operating at low-power $(40-80 \mathrm{~W})$ with this application in mind. The ECR waveguide plasma source that has been developed at the U-M Plasma Science and Technology Laboratory is described, along with the basis for its design. This plasma source eliminates any lifetime limitations in more conventional devices, because it uses traveling microwaves within a waveguide to heat the discharge. Microwaves are launched by an antenna within a coax-waveguide transition, which is isolated from the discharge by a pressure window, thereby preventing any direct contact between the antenna and the plasma. The source uses permanent magnets to establish resonance heating zones, and should be scalable to various sizes depending on the microwave frequency being used. The waveguide configuration also has the capability of handling high microwave power levels if necessary, without requiring microwave antenna cooling. In some ECR plasmas, the electrons within the discharge are so strongly magnetized that they cannot easily be extracted from the source, so several magnetic circuits were tested with the waveguide plasma source. Some experimental data regarding the relevant plasma parameters and the amount of extractable electron current from the source are also presented in this paper. The limitations of the source are identified, and the next steps to be taken to optimize the performance of the source are explained.

\section{Device Description}

A cross-sectional drawing of the waveguide plasma source is shown in Fig. 1. The body of the source is made of brass, and the gas feedthrough is located $5.0 \mathrm{~cm}$ from the exit plane of the source. The source body is silver soldered to two mounting flanges. The larger bottom flange mates with the vacuum chamber cover with a Teflon spacer in between the two, which will allow for independent DC biasing of the source body in future tests. The other flange mates with a quartz pressure window, with the coax-to-circular waveguide transition on the other side 
of the window. This allows for the antenna to be completely separated from the plasma production region. Additionally, there are two circular bolt patterns on the bottom of the source, which will be used in future testing to mount orifice and keeper electrodes. Each of these can be isolated with the use of insulating bolts and spacers. Figure 2 shows a photograph of the source with the gas feedthrough, pressure window, and coax transition included.

The source is $17.6 \mathrm{~cm}$ long, and it includes two rectangular slots situated across from one another, which are large enough to hold two SmCo magnets each. The waveguide tube also contains two oppositely positioned triangular protrusions that were oriented in the plane perpendicular to that of the magnet slots, and parallel to the polarization direction of the $\mathrm{TE}_{11}$ mode driven by the coax-to-waveguide adapter (not shown). The purpose of the magnet slots and the triangular electric field enhancing protrusions is to force the ECR heating zone to be situated at the center of the tube.

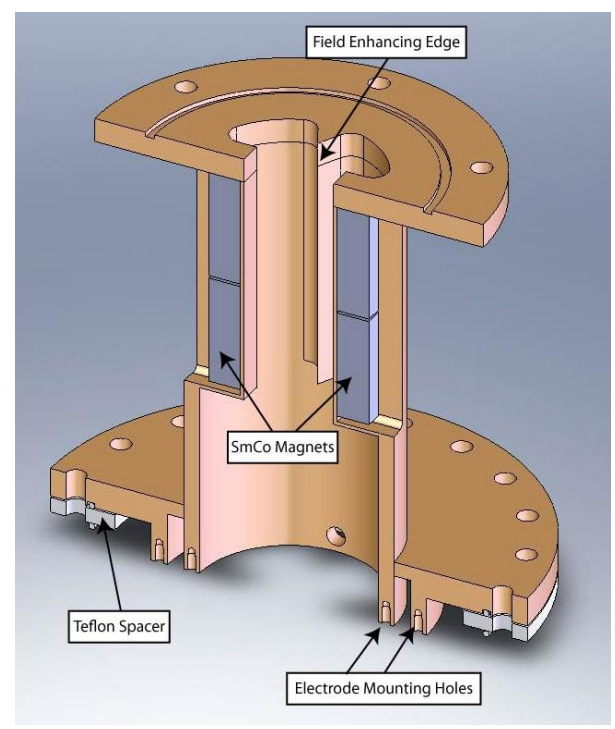

Figure 1. Cross-section drawing of the source with magnets and Teflon spacer.

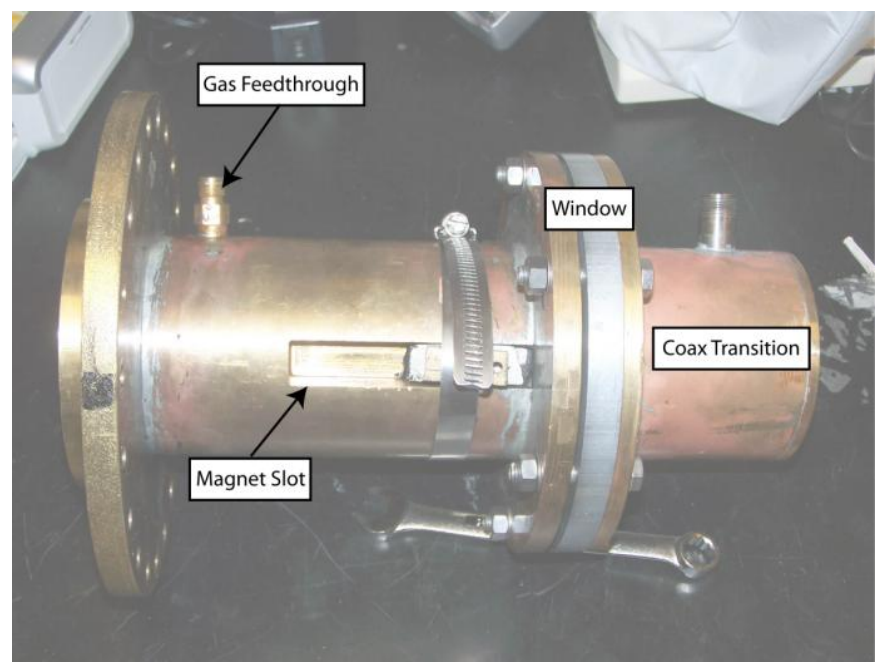

Figure 2. Photograph of plasma source with window, gas feed, and coax transition. 


\section{Design Basis}

Initially, the ECR waveguide plasma source was intended to be a simple cylindrical waveguide under vacuum, with samarium cobalt magnets situated around the outside of the waveguide in order to set up the ECR plasma production zones. The desired current would then be extracted from the end of the waveguide by a biased electrode or grid. The final design of the prototype source, however, turned out to be quite different from this initial layout. The prototype design was developed through the use of finite element electromagnetic and magnetostatic field solvers.

\section{A. Cutoff Calculations}

In order to separate the microwave antenna from the bulk plasma and prevent erosion of the antenna by sputtering, the plasma within the source is generated by traveling microwaves, as opposed to an electric field oscillating at the microwave frequency produced by an immersed antenna. This naturally places a size constraint on the plasma source, since the frequency of the microwaves propagating in a given $\mathrm{TE}_{\mathrm{nm}}$ mode must be higher than the cutoff frequency of the mode, which is determined by the dimensions of the waveguide or cavity. In other words, for a given $\mathrm{TE}_{\mathrm{nm}}$ mode at a fixed frequency in a cylindrical waveguide, the waveguide radius must be larger than the cutoff radius for that mode, given by, ${ }^{13}$

$$
a_{c, n m}=\frac{p_{n m}^{\prime}}{2 \pi f \sqrt{\mu \varepsilon}}
$$

where $a_{c, n m}$ is the cutoff radius for mode $\mathrm{TE}_{\mathrm{nm}}, f$ is the microwave frequency, $\mu$ and $\varepsilon$ are the permeability and permittivity, respectively, of the medium within the waveguide, $p_{n m}{ }_{n m}$ is the $m$ th root of $J_{n}$ ', and $J_{n}{ }^{\prime}$ is the derivative of the $n$th Bessel function of the first kind. A similar relation gives the cutoff radius for the $\mathrm{TM}_{\mathrm{nm}}$ modes at a fixed microwave frequency:

$$
a_{c, n m}=\frac{p_{n m}}{2 \pi f \sqrt{\mu \varepsilon}}
$$

where, $p_{n m}$ is the $m$ th root of $J_{n}$, and $J_{n}$ is just the $n$th Bessel function of the first kind.

Equations (1) and (2) show that for $2.45 \mathrm{GHz}$ microwaves, the frequency used in this study, the cutoff radius in vacuum is $4.69 \mathrm{~cm}$ for the $\mathrm{TM}_{01}$ mode, 3.59 for the $\mathrm{TE}_{11}$ mode, and larger than both of these values for all other modes. In order to prevent mode competition within the plasma source, it was designed to operate in only the $\mathrm{TE}_{11}$ mode with an inner waveguide radius of $3.8 \mathrm{~cm}$.

\section{B. Magnetic Circuit Design}

The electric field pattern of the circular $\mathrm{TE}_{11}$ mode in a cylindrical waveguide is shown in Fig. $3 .{ }^{13}$ It is clear that the electric field strength in this mode is strongest at the center of the waveguide and weakest near the walls. In order to produce a discharge by ECR or upper hybrid heating, it is necessary to have the electric field perpendicular to the applied magnetic field. When using microwaves at $2.45 \mathrm{GHz}$, these heating mechanisms take place when the applied magnetic field strength is near 875 Gauss. In the waveguide plasma source, it is desirable to set up this ECR zone near the center of the waveguide, so that it overlaps the region of strongest electric field.

Ansoft Maxwell 2D was used to set up a magnetostatic model of the plasma source with several magnetic geometries. The goal of these simulations was to determine the best location for the permanent magnets to set up the ECR zone on axis. Four example field plots are shown in Fig. 4. The examples on the left show a configuration where the permanent magnets are placed against the inner wall of a circular waveguide, and the magnets are in attraction with one another. This type of configuration would be simple to construct, by just adhering the magnets to the waveguide itself. The

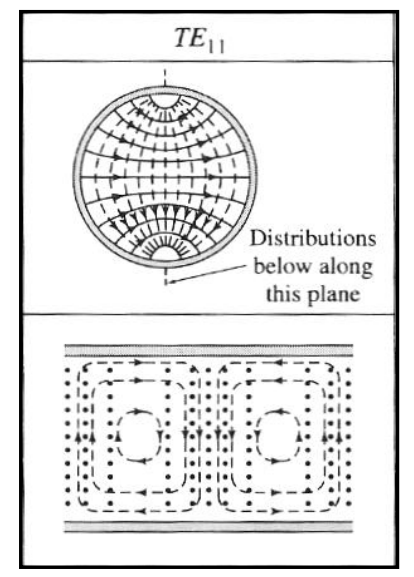

Figure 3. Circular TE11 mode field pattern. Solid lines represent electric field, and dashed lines represent magnetic field. 
plots on the right show a different configuration, a waveguide that is modified with two rectangular slots. This "slotted" geometry allows for the magnets to be placed outside the waveguide, in attraction with one another.

The slotted tube geometry allows for the resonance zone to be established on axis when the magnets are in attraction. In the configurations shown in Fig. 4, the static magnetic field vectors are perpendicular to the microwave electric field vectors shown in Fig. 3. In addition, the microwave propagation vector is oriented axially, so that the $\mathbf{E}, \mathbf{B}$, and $\mathbf{k}$ vectors are mutually perpendicular on centerline. The electromagnetic wave in a plasma which has these vector orientations is known as the extraordinary wave (X-wave). The high-frequency resonance of the $\mathrm{X}$-wave is the upper hybrid frequency, and the plasma will fully absorb the incident microwaves at the resonance condition,

$$
\omega^{2}=\omega_{U H}^{2}=\omega_{p e}^{2}+\omega_{c}^{2}
$$

When the magnets are in attraction within the slotted tube geometry shown in Fig. 4, the dominant method of microwave absorption should be upper hybrid heating, based on the orientations of $\mathbf{E}, \mathbf{B}$, and $\mathbf{k}$. Therefore, Eq. (3) can be used in this case to relate the plasma, cyclotron, and microwave frequencies at the heating zones.
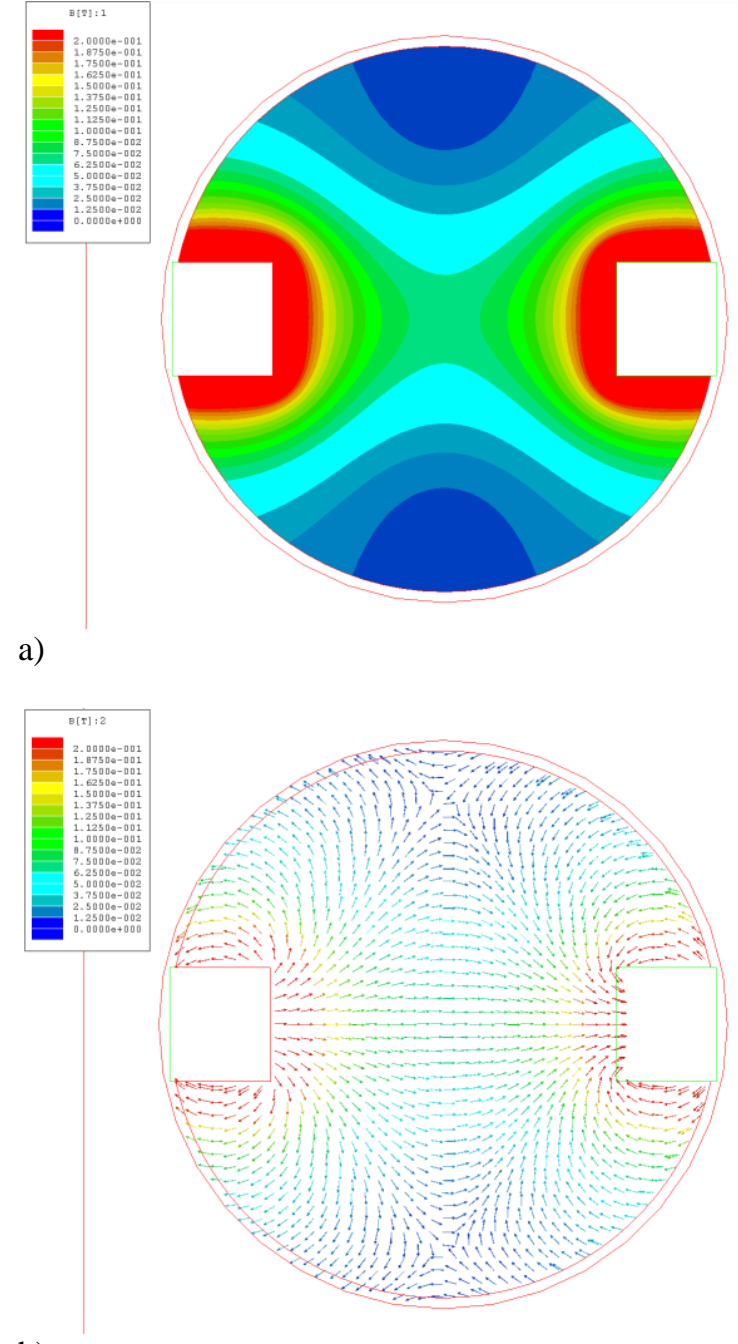

b)

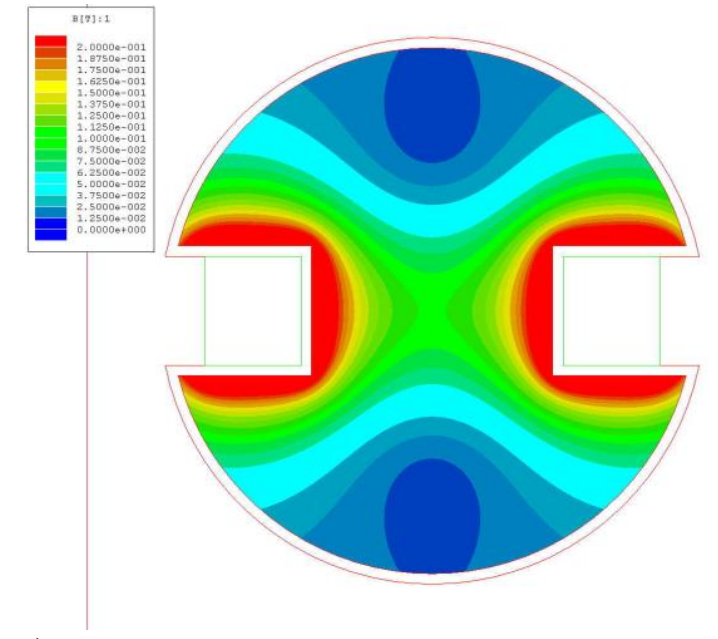

c)

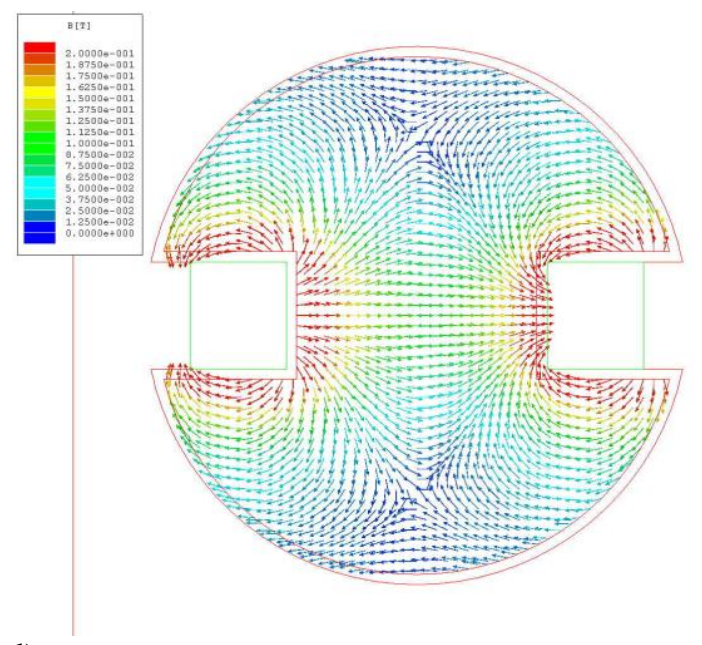

d)

Figure 4. Magnetic field contour (a) and vector (b) plot of simple circular waveguide geometry, with magnets placed in attraction on the inner wall of the tube. Contour (c) and vector (d) plot of slotted waveguide geometry, with magnets placed in attraction, on the outside of the tube.

When the magnets are in repulsion in this slotted tube geometry, the 875 Gauss zone is pushed slightly off-axis, as shown in Fig. 5. There is a field-free zone on centerline, which should facilitate electron transport along the axis 
of the tube. The 2D model only shows the magnetic field vectors being directed outward toward the walls of the tube, but in the actual 3D case with finite length magnets, the repelling magnets set up an axial magnetic field just outside the field-free region. In this case, $\mathbf{B}$ is perpendicular to $\mathbf{E}$ but parallel to $\mathbf{k}$ near the axis. These vector orientations allow for the propagation of right-hand and left-hand circularly polarized (RHCP and LHCP) waves in a plasma. The RHCP wave produces a rotating electric field vector which can synchronize with the electron orbit around the magnetic field lines at the ECR condition,

$$
\omega^{2}=\omega_{c}^{2}
$$

In regions where Eq. (4) holds, ECR heating will take place, and the plasma density that can be obtained from this mechanism has no theoretical limit. A desirable ECR plasma source will produce an "overdense" plasma, which has a plasma frequency greater than or equal to the microwave frequency:

$$
\omega^{2} \leq \omega_{p e}^{2}=\frac{n e^{2}}{m \varepsilon_{0}}
$$

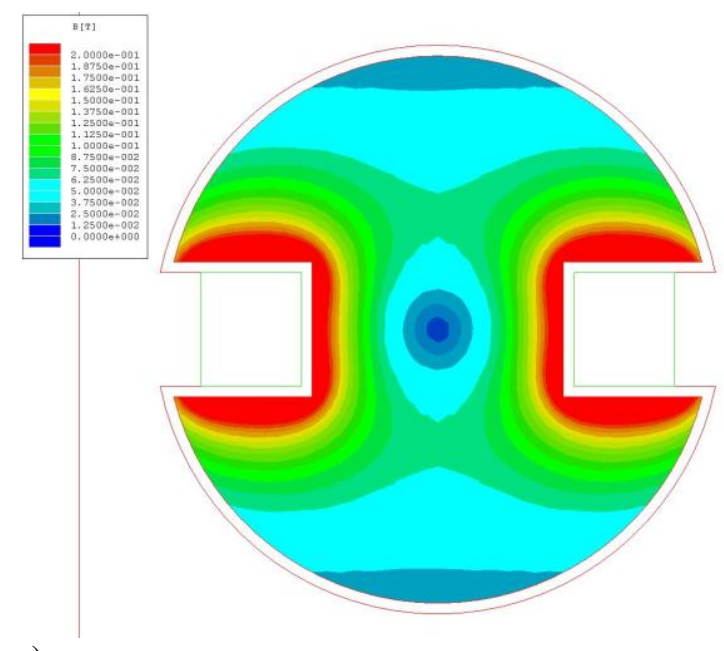

a)

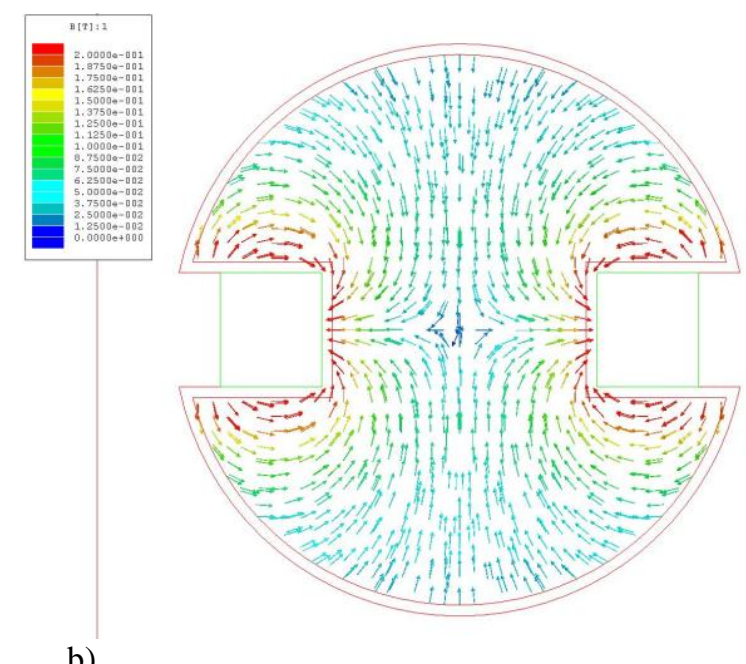

b)

\section{Figure 5. Magnetic field contour (a) and vector (b) plots of slotted waveguide geometry, with bar magnets placed in repulsion.}

For more discussion on electromagnetic waves and particle motions in plasmas, the reader is directed to Reference 15. In reality, both magnetic circuits have some degree of magnetic field curvature, so both types of heating are expected within the source to an extent. The slotted tube design appeared to be an attractive means of setting up the heating zones, so it was incorporated into the design of the source.

\section{Microwave Circuit Design}

After the slotted cylindrical waveguide design was chosen for the magnetic circuit, a model of the waveguide structure was developed in Ansoft HFSS. The model was used to determine the microwave electric field pattern within the waveguide, and to finalize the layout of the source. In the simulation, one port corresponding to the pressure window above the source was set as the launching port for incident microwaves, driven at $2.45 \mathrm{GHz}$ at a power level of 1 Watt. The other end of the waveguide was capped off with a cover plate which contained an orifice of diameter $5.0 \mathrm{~mm}$ and length $1.5 \mathrm{~mm}$. The orifice was specified as the "exit port," for the microwaves, which were polarized in the direction perpendicular to the plane of the magnet slots.

First, a model of the geometry shown in Figs. 4 (c) and (d) was run, and the resulting distribution of the electric field magnitude was plotted. The electric field perpendicular to the slots was nearly an order of magnitude smaller than the field across the slots, and so triangular field enhancing edges were introduced in the direction of polarization. Additionally, it was determined that the presence of the orifice plate on the end of the waveguide 
resulted in a fixed axial distribution of electric field strength. As expected, the $\mathrm{TE}_{11}$ mode electric field was zero at the end plate, but the peak electric field strength occurred roughly $10 \mathrm{~cm}$ from the orifice plate. The slots were then modified so that they were localized to this region of strong electric field. After several variations of the model, a nearly final design was reached, with the magnet slots located from 8.1 to $18.2 \mathrm{~cm}$ from the orifice plate. The results of the simulation, shown in Fig. 6, demonstrate the improvement of the electric field strength between the localized magnet slots and field enhancers.
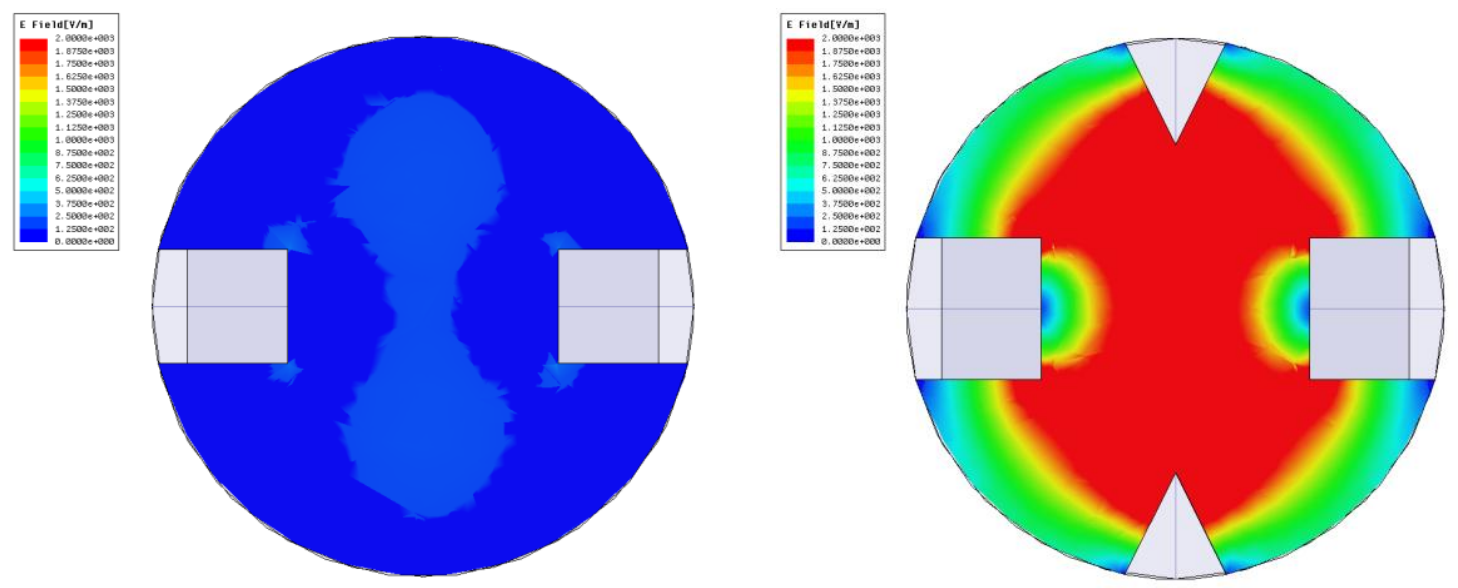

Figure 6. Microwave electric field strength with (left) and without (right) field enhancers.

The edges in the design were eventually rounded, due to machining complications, but the electric field strength increased along the entire length of the magnet slots because of this. The field electric field patterns of the final design are shown in Fig. 7. Wall currents associated with the microwave propagation were also calculated to determine the best location for the gas feed port. The gas feedthrough was installed at a location directly beneath the field enhancers where little to no surface current flowed so as to minimize its perturbation to microwave propagation. 


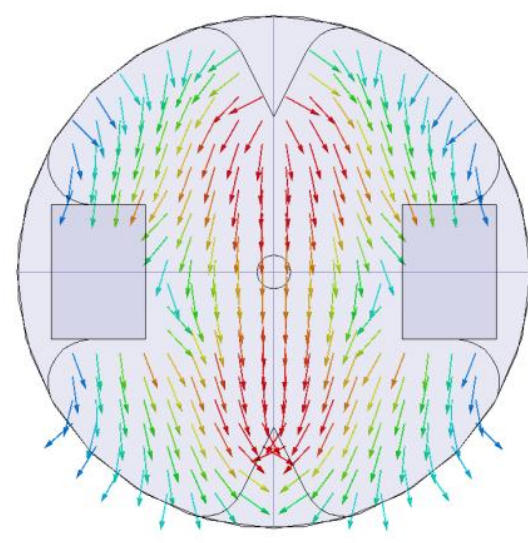

a)

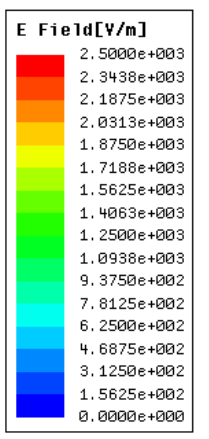

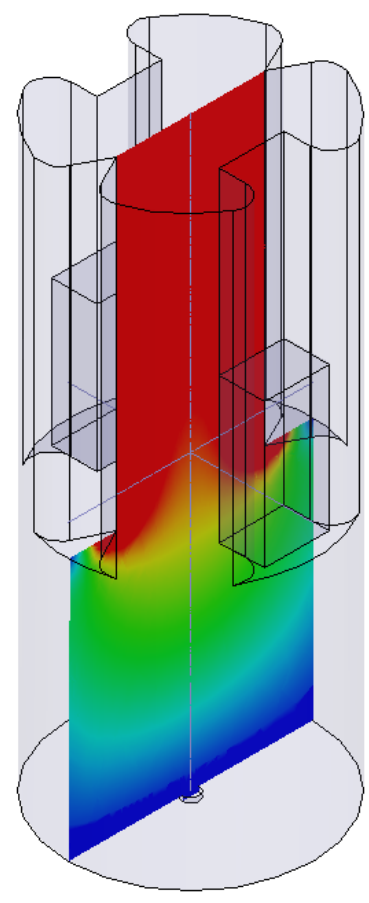

c)
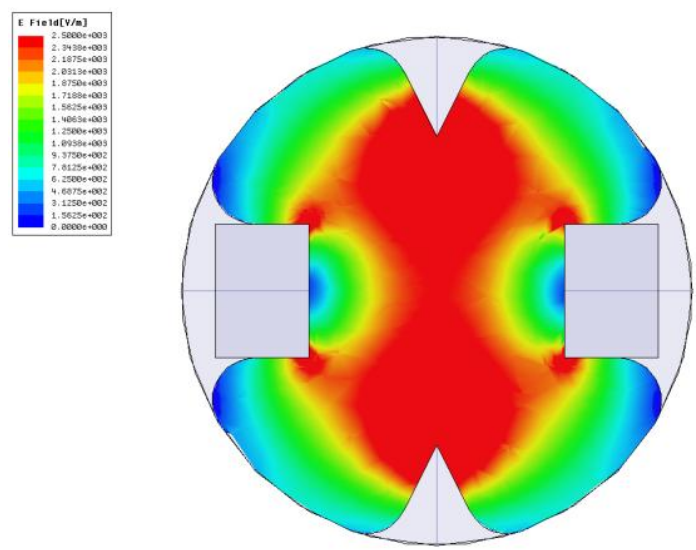

b)
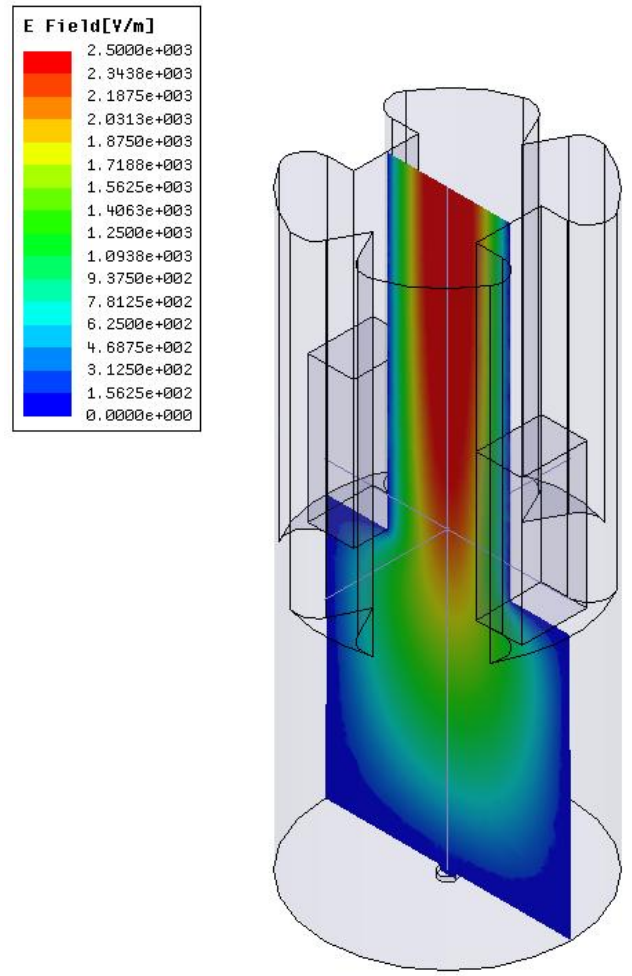

d)

Figure 7. Plots of microwave electric field in final design. Vector (a) and contour (b) plots of electric field in the top view, with contour plots in the planes parallel (c) and perpendicular (d) to field enhancers.

\section{Three-dimensional Magnetostatic Calculations}

When the waveguide design was finalized, Ansoft Maxwell 11 was used to carry out a full three-dimensional simulation of the static magnetic field pattern due to the SmCo magnets within the slots. The calculation confirmed the Maxwell 2D results showing the 875 Gauss ECR zone near the axis of the tube, while also generating an axial view of the magnetic field profile. In addition, the calculated magnetic field vectors were broken into components and stored in a matrix of $1 \mathrm{~mm}$ elements, for possible future use in PIC calculations of plasma diffusion within the source. The axial profile of the magnetic field is shown in Fig. 8. 


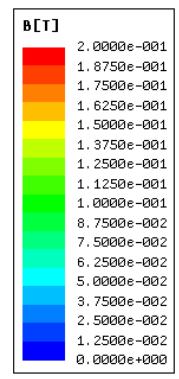

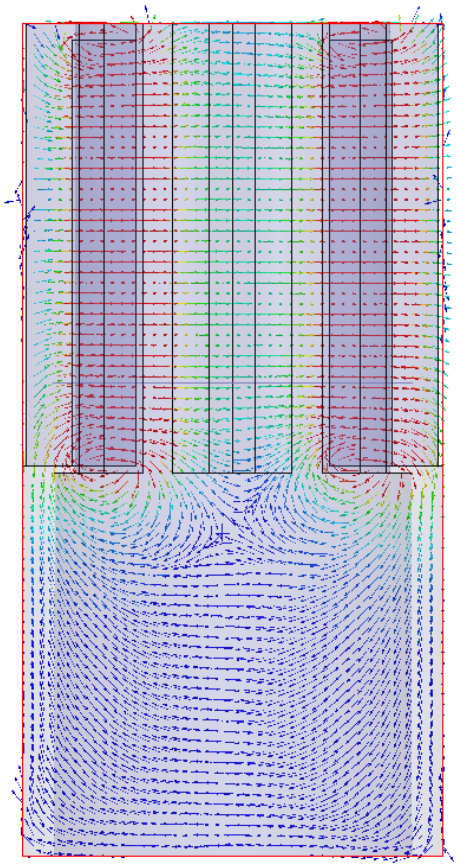

a)
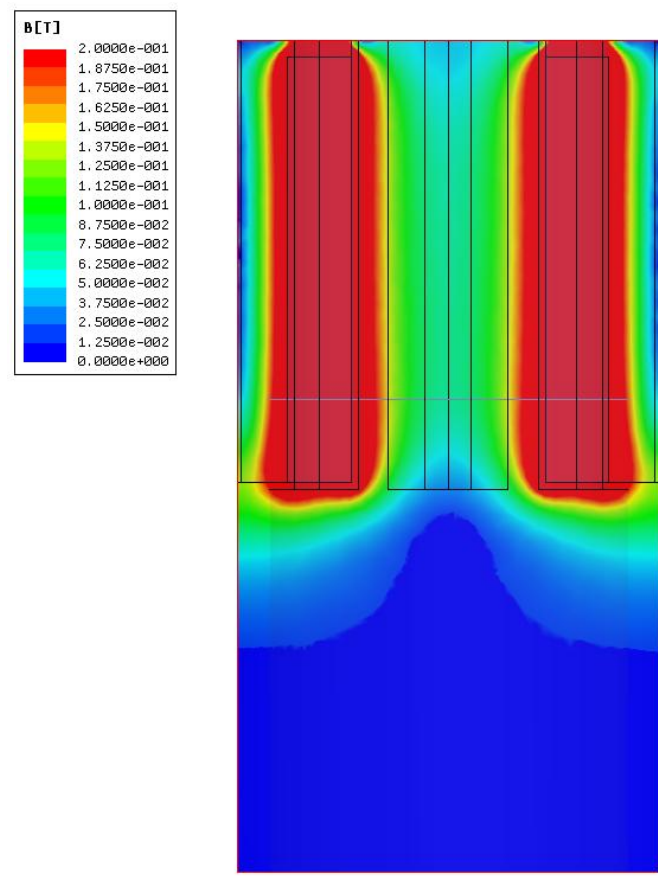

b)

Figure 8. Axial profile of the magnetic field, shown in vector (a) and contour (b) plots in the plane through the center of the magnet slots.

\section{Testing Facility Description}

For testing, the waveguide plasma source was mounted, on axis, to the top of a vacuum chamber with an inner diameter of $45 \mathrm{~cm}$ and a length of $62 \mathrm{~cm}$. The exit plane of the plasma source was oriented downward into the chamber. The chamber itself was evacuated by a Pfeiffer L 80 rotary vane pump through a Pfeiffer-Balzers TPU 170 turbomolecular pump, with a pumping speed of $170 \mathrm{~L} / \mathrm{s}$ on $\mathrm{N}_{2}$. The argon gas flow into the plasma source was controlled by a Vacoa MV-25 leak valve, and the gas flow rate was measured by an Omega FMA 1802 flow meter, which has a range of $0-10 \mathrm{sccm} \mathrm{N}_{2}$. Pressure in the vacuum chamber was monitored with a Lesker KJL-6000 thermocouple and G100F ion gauge, with a KJL 4500 ion gauge controller. A photograph of the vacuum facility is shown in Fig. 9.

Microwaves are launched into the other end of the waveguide plasma source by a coax to circular $\mathrm{TE}_{11}$ mode transition, through a Tensolite WHU 18-1818 microwave cable. The rest of the microwave circuit consists of WR340 waveguide components. A MH2.0W-S magnetron head from National Electronics is powered by a Alter SM 7456 power supply unit, and it is directly attached to a National Electronics D0716 circulator. The circulator is attached to a 2-way, $60 \mathrm{~dB}$

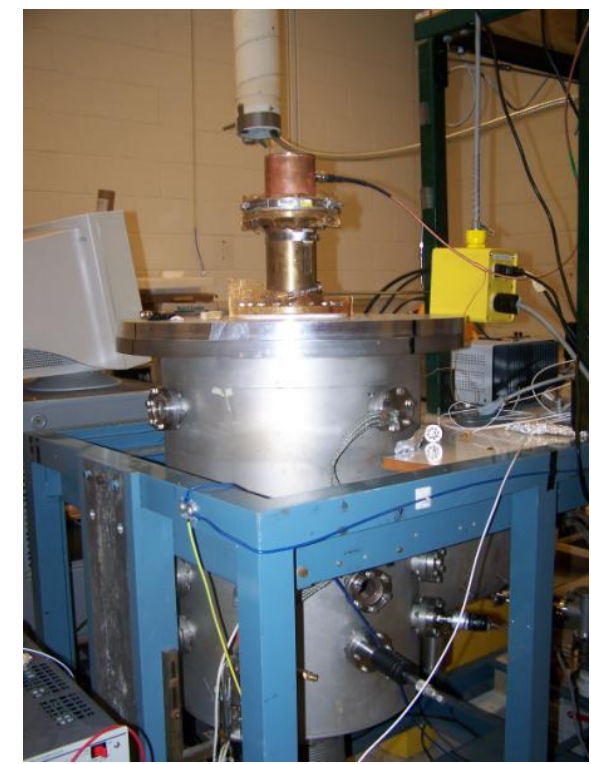

Figure 9. Photograph of the vacuum facility. directional coupler followed by a Sariem 3-stub tuner and a WR340-to-coax transition, also by National Electronics. The forward and reflected power levels were monitored by HP435B Power Meters. The microwave setup is shown in Fig. 10. 


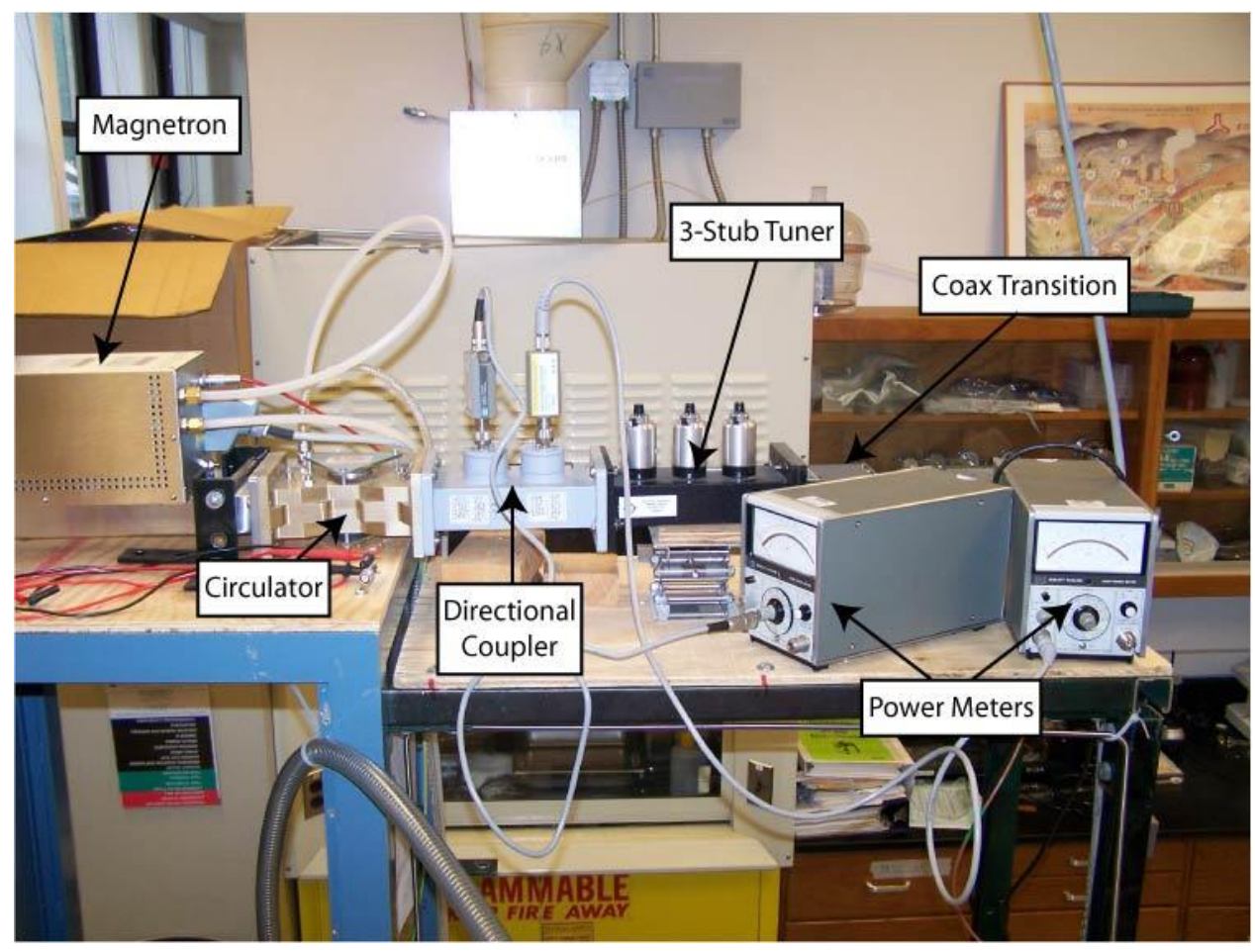

Figure 10. Photograph of the microwave setup.

For initial testing of the source, a simple circuit was set up to extract electron or ion current from the plasma source. A circular steel electrode, with a diameter of $5.6 \mathrm{~cm}$ and a thickness of roughly $1 \mathrm{~mm}$, was attached to the brass waveguide by a mounting bracket. The electrode was isolated from the rest of the setup by a ceramic standoff, and it was connected to an electrical feedthrough by Teflon-coated wire. A variable bias voltage was applied to the electrode by a GW Instek 60 Volt, 3 Ampere power supply unit, while the extracted current was measured by a Fluke $87 \mathrm{~V}$ multimeter. Another Fluke $87 \mathrm{~V}$ multimeter was used to measure the floating potential of the extraction electrode, and the power supply voltage was verified by a Fluke 115 multimeter. To ensure that the microwave cable would not overheat, a thermocouple wire was attached to the cable and the temperature was monitored with a Omega HH12A thermocouple meter.

In another test, Langmuir probes were used to characterize the plasma within the source. The Langmuir probe traces were recorded using the SmartProbe driver by Scientific Systems. Two probes were constructed from .508 $\mathrm{mm}$ diameter molybdenum wire, and each probe tip had a length of $7.0 \mathrm{~mm}$. Both probe wires were fed through a single piece of double-bore alumina tubing, with one probe tip oriented axially, and the other probe tip oriented perpendicular to the axis of the waveguide. With each probe, the SmartProbe software automatically swept the probe bias voltage and recorded the resulting probe current. The software calculated the electron temperature and density, floating potential, and plasma potential from each probe trace. A picture of the probe assembly is shown in Fig. 11. 


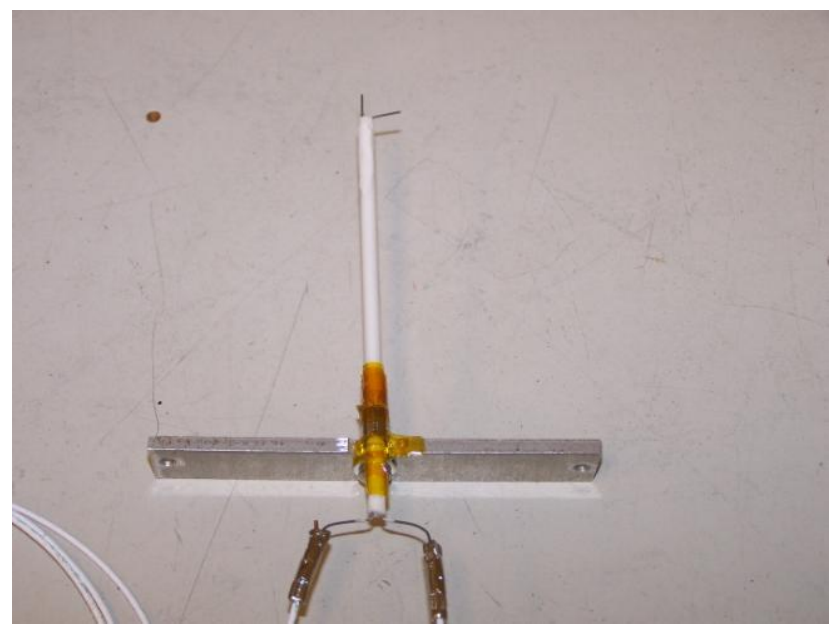

Figure 11. Photograph of Langmuir probe assembly.

\section{Experimental Data}

\section{A. Preliminary Testing}

The objective of the first tests with the waveguide plasma source was to determine the static magnetic field geometry within the slots that would enable the most electron current to be extracted from the discharge. Several magnet configurations within the slots were tested, as illustrated in Fig. 12.

In each configuration, the absorbed power level was fixed at $60 \mathrm{~W}$, and the gas flow rate was varied from 0 to 15 $\mathrm{sccm}$ of argon. The electrode was placed in the exit plane of the source, on axis. It was biased at $+40 \mathrm{~V}$ during the sweep, and the maximum amount of extracted current was recorded along with the corresponding flow rate. Then, the electrode was also biased at $-40 \mathrm{~V}$ and the test was repeated. In this case, the maximum extracted ion current and corresponding flow rate were recorded. The results of all of these tests are compiled in Table 1.

During testing, it was observed that in all cases when the magnets were placed in attraction across the slots, the discharge self-started at relatively low power levels of 25-30 W. The discharges appeared to be stable as there was little to no variation in the current readings after repeated measurements. For the cases with the magnets placed in attraction, the maximum extractable current corresponded to flow rates around $1.5-4 \mathrm{sccm}$.

When the magnets were placed in repulsion across the slots, the source behaved quite differently. Initially, the assumption was that when the magnets are placed in repulsion, the electrons within the production region would not be trapped by the strong magnetic field. Rather, the strongly magnetized electrons would follow the field lines out of the plasma production region and toward the extraction electrode, since the field lines in would be oriented axially and radially outward from this region. The partially axial magnetic field set up by the repelling magnets would also allow for pure ECR heating, in contrast with upper hybrid heating.

In all cases involving repelling magnets but one, the source would not self start unless additional magnets were initially placed around the outside of the source for confinement. This was done by literally placing a magnet near the outside of the source so that ECR coupling could occur internally. This is all possible because the outside body of the source was at atmospheric pressure as indicated in the experimental set-up photograph. Also, the discharge appeared more unstable, as it would flicker and occasionally extinguish. In Case 5, the discharge could not be sustained unless the magnets used to start the discharge were permanently held in place.

After compiling the data in Table 1, it was determined that further optimization of the source should start with the magnets placed in attraction across the slots. While Case 4 was able to extract $131 \mathrm{~mA}$ of electron current, this corresponded to a flow rate of $15.8 \mathrm{sccm}$, which is unreasonably large for a neutralizer source. Additionally, the instability that was observed with the slots in repulsion raised concerns about how sensitive source operation would be to discharge adjustments. The instability was assumed to be related to the fact that when the slots are in repulsion, electrons are not confined as well as when the slots are in attraction. The magnetic field lines are directly incident on the walls and the extraction electrode in this case, and since the electrons move freely along the field lines, they are not confined well enough to generate sufficient ionization to sustain the discharge. 


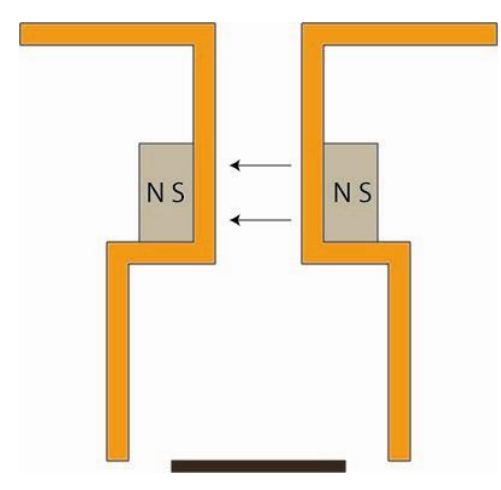

a) "Case 1"

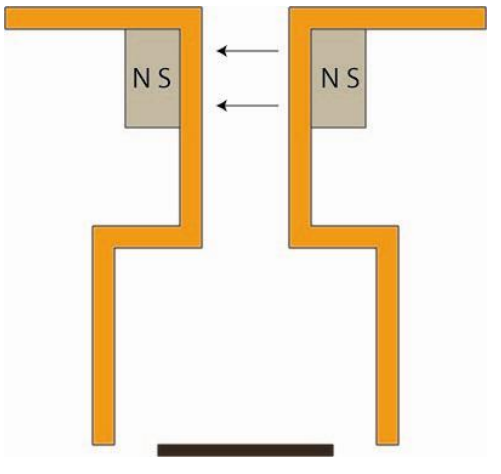

b) "Case 2"

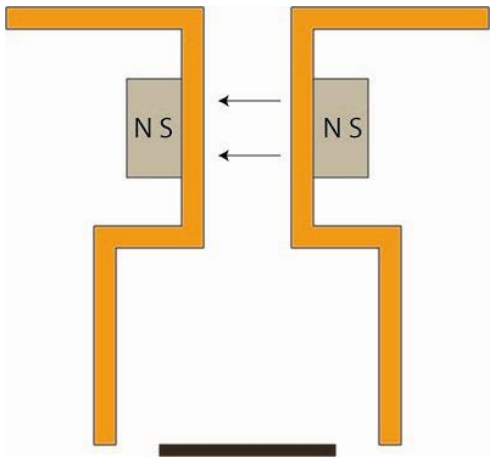

c) "Case 3"

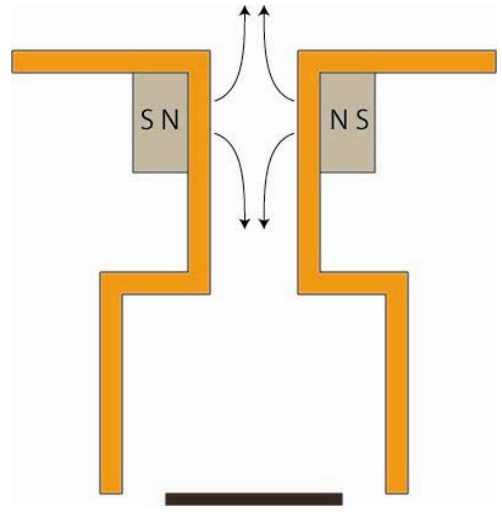

d) "Case 4"

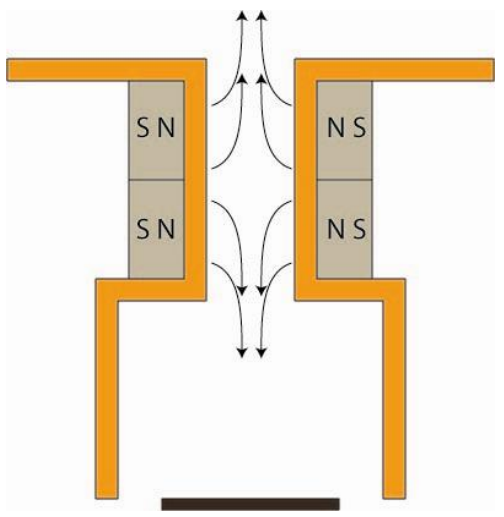

f) "Case 6"

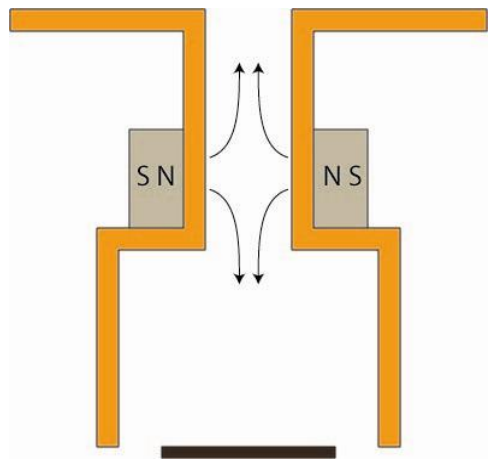

e) "Case 5"

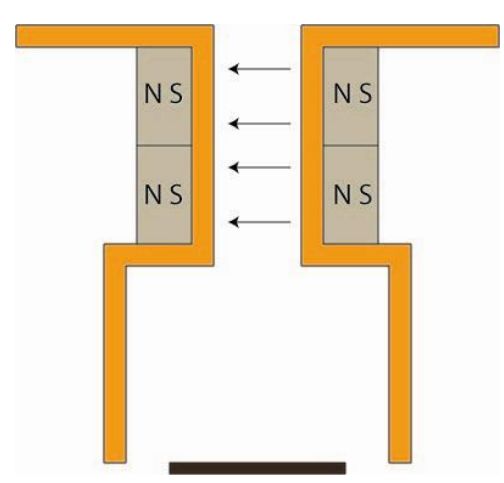

g) "Case 7"

Figure 12. Magnetic configurations investigated in preliminary testing. 
With the slots in attraction, the electrons are confined to the field lines, which go directly from one slot to the other. At the surface of the slots, the electrons can be reflected back into the plasma by the magnetic cusp, resulting in further ionization and a more stable discharge. In these configurations (Cases 1-3 and 6) the extractable current is highest at low flow rates, so the peak utilization of the feed gas is larger than in Cases 4 and 6.

Table 1. Extracted current as a function of magnetic configuration.

\begin{tabular}{|c|c|c|c|c|c|}
\hline Case\# & Slot Configuration & $\begin{array}{l}\text { Max Electron } \\
\text { Current [mA] }\end{array}$ & $\begin{array}{l}\text { Max e-Flow } \\
\text { Rate [sccm] }\end{array}$ & $\begin{array}{c}\text { Max lon } \\
\text { Current }[\mathrm{mA}]\end{array}$ & $\begin{array}{l}\text { Max I+ Flow } \\
\text { Rate [sccm] }\end{array}$ \\
\hline 1 & $\begin{array}{c}1 \text { magnet each, } \\
\text { bottom of slots, } \\
\text { attraction }\end{array}$ & 44.4 & 3.8 & 1.5 & 1.5 \\
\hline 2 & $\begin{array}{l}1 \text { magnet each, top } \\
\text { of slots, attraction }\end{array}$ & 75.6 & 2.0 & 1.5 & 1.3 \\
\hline 3 & $\begin{array}{l}1 \text { magnet each, } \\
\text { centered in slots, } \\
\text { attraction }\end{array}$ & 48.6 & 3.4 & 1.1 & 1.5 \\
\hline 4 & $\begin{array}{l}1 \text { magnet each, top } \\
\text { of slots, repulsion }\end{array}$ & 131.0 & 15.8 & 0.7 & 3.9 \\
\hline 5 & $\begin{array}{l}1 \text { magnet each, } \\
\text { bottom of slots, } \\
\text { repulsion }\end{array}$ & \multicolumn{4}{|c|}{ No Discharge } \\
\hline 6 & $\begin{array}{l}2 \text { magnets each, } \\
\text { repulsion }\end{array}$ & 38.4 & 15.8 & 0.8 & 3.6 \\
\hline 7 & $\begin{array}{l}2 \text { magnets each, } \\
\text { attraction }\end{array}$ & 57.3 & 1.3 & 2.0 & 1.3 \\
\hline
\end{tabular}

\section{B. Current Extraction Tests - Two Magnets per Slot}

For a more thorough study of the extractable electron current in the source, two magnets were placed in each slot, with the magnets in attraction across the two slots. The source was operated in this way with three different electrode configurations, as shown in Fig. 13. In each test, the gas flow rate was swept from 0.7 to $14.6 \mathrm{sccm}$, at power levels of 40,60 , and $80 \mathrm{~W}$. At each flow rate, the extracted electron and ion current at electrode biases of +60 and $-60 \mathrm{~V}$, respectively, were recorded along with the floating potential of the electrode. In the first test, Setup A, the extraction electrode was placed $0.7 \mathrm{~cm}$ below the magnet slots inside the tube. In Setup B, the electrode was placed at the exit plane of the source, $8.1 \mathrm{~cm}$ below the magnet slots. In Setup C, the electrode was at the exit plane of the source and a mica sleeve was placed around the walls of the source below the slots. The purpose of the mica was to prevent plasma losses to the wall in that area. In all three setups, the source easily self-started at absorbed power levels around $25 \mathrm{~W}$. 


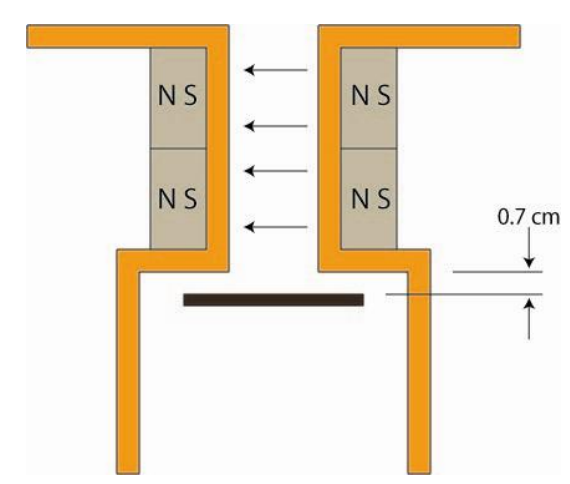

a) "Setup A"

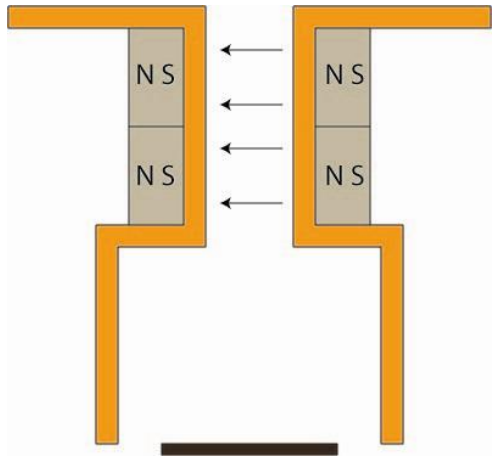

b) "Setup B"

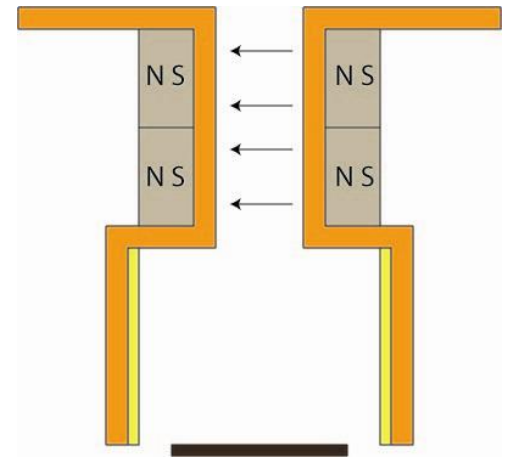

c) "Setup C"

Figure 13. Electrode configurations investigated during current extraction tests with 2 magnets per slot.

A comparison of the extracted electron current as a function of flow rate is shown in Fig. 14 for the three configurations. The most noticeable feature of the plot is that the current in Setup A is significantly larger than in Setups B and C over the entire range of flow rates. This makes intuitive sense, since the magnetic field between the slots is on the order of 800 Gauss and higher. With the magnets in attraction, the electrons within the discharge must diffuse directly across the field lines to reach the electrode in all setups. The classical diffusion coefficient in a magnetic field is given by,

$$
D_{\perp}=\frac{D}{1+\omega_{c}^{2} \tau^{2}}
$$

Where $D_{\perp}$ is the diffusion coefficient across a magnetic field, $D$ is the non-magnetized classical diffusion coefficient, $\omega_{c}$ is the cyclotron frequency, and $\tau$ is the mean collision time. For electrons in a strong magnetic field, $\left(\omega_{c} \tau\right)^{2} \gg 1$, so the diffusion coefficient for electrons scales as $1 / B^{2}$, where $B$ is the magnetic field strength. One consequence of this is that while the electrons are well-confined and can produce a stable discharge, they are essentially trapped between the magnet slots. In Setup A, the electrode is close enough to the magnets that some field lines terminate directly on the extraction electrode, allowing electrons to be collected. In Setups B and C, where the magnetic field is negligible at the electrode, the electrons cannot easily diffuse across the field lines to reach the electrode.

Another interesting point is that the difference in extracted current between Setups B and C is relatively small, and quite negligible over a range of flow rates. In any plasma cathode, the electron current extracted by the electrode must equal the ion current lost to the walls of the source to prevent charge buildup. The fact that Setups B and $\mathrm{C}$ are so similar suggests that the lower section of the source does not contribute appreciably to plasma particle collection. In other words it makes little difference whether the lower section is exposed or isolated with the mica. 


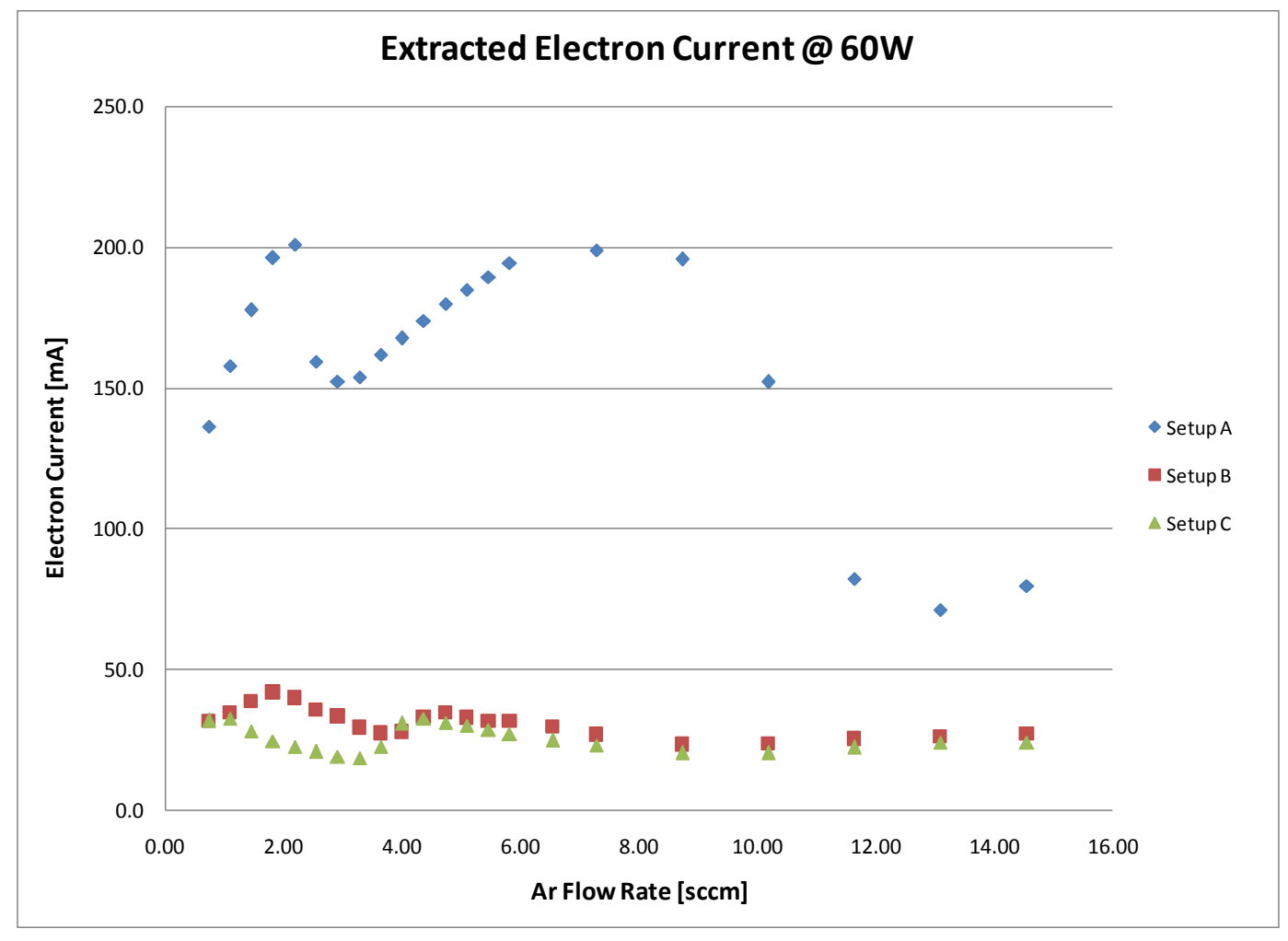

Figure 14. Comparison of extracted electron current in Setups A, B, and C.

The plot in Fig. 15 shows the extracted current as a function of flow rate and absorbed power, for Setup A only. Also included in the plots is the $71.7 \mathrm{~mA} / \mathrm{sccm}$ line, which corresponds to one electron being extracted per neutral fed into the source. The extracted current is larger than this value at flow rates between 1 and $2.5 \mathrm{sccm}$, depending on the power level. At low flow rates, some ions recombine at the source wall and are subsequently reionized, contributing additional electrons to the extracted current. With the help of this ion recycling, the source was able to extract $226 \mathrm{~mA}$ at $80 \mathrm{~W}, 2.6 \mathrm{sccm}$; and $201 \mathrm{~mA}$ at $60 \mathrm{~W}, 2.2 \mathrm{sccm}$. Clearly the source is most efficient at electron production the low flow rates. This is somewhat puzzling in that higher flow rates tend to enhance electron cross field diffusion. One would expect based on classical diffusion that the extracted electron current should increase with flow rate. Such trends are seen as local peaks at higher flows but the associated current is well below the reference line $(71.7 \mathrm{~mA} / \mathrm{sccm})$.

At 40 and 60 Watts, there are two distinct peaks in the extracted current level: one corresponding to the range where ion recycling takes place and the other at higher flow rates. The presence of the peaks appearing at higher flow rates may be due to a shift in the position of the ECR heating zone. Such a shift is related to the fact that absorption of the microwaves is chiefly at the upper hybrid resonance. As plasma density increases with flow rate, the absorption zone shifts to regions of weaker magnetic field strength. Drop-off in current after it peaks may be due to the shifting of the plasma production toward centerline where the magnetic field is weaker. The reduced plasma production volume would then give rise to a drop in density with flow rate as observed here. Secondary peaks are likely consequences of absorption taking place at more favorable locations, perhaps those regions further downstream where fringing is appreciable. This suggests that a more optimal configuration would be one in which the transverse field decreased in magnitude axially so that as density increased hybrid absorption at fixed frequency can take place in the weaker field regions. At a given power, the peaks shift to higher flow rates as the absorbed power increases. It is expected that as the flow rate increases, so does the pressure, and the plasma density will increase as long as the ionization rate constant multiplied by the gas density is increasing. With increasing pressure, the mean average energy of the electron, due to a decreased number of cyclotron cycles between collisions, decreases resulting in a reduction in overall ionization rate. 


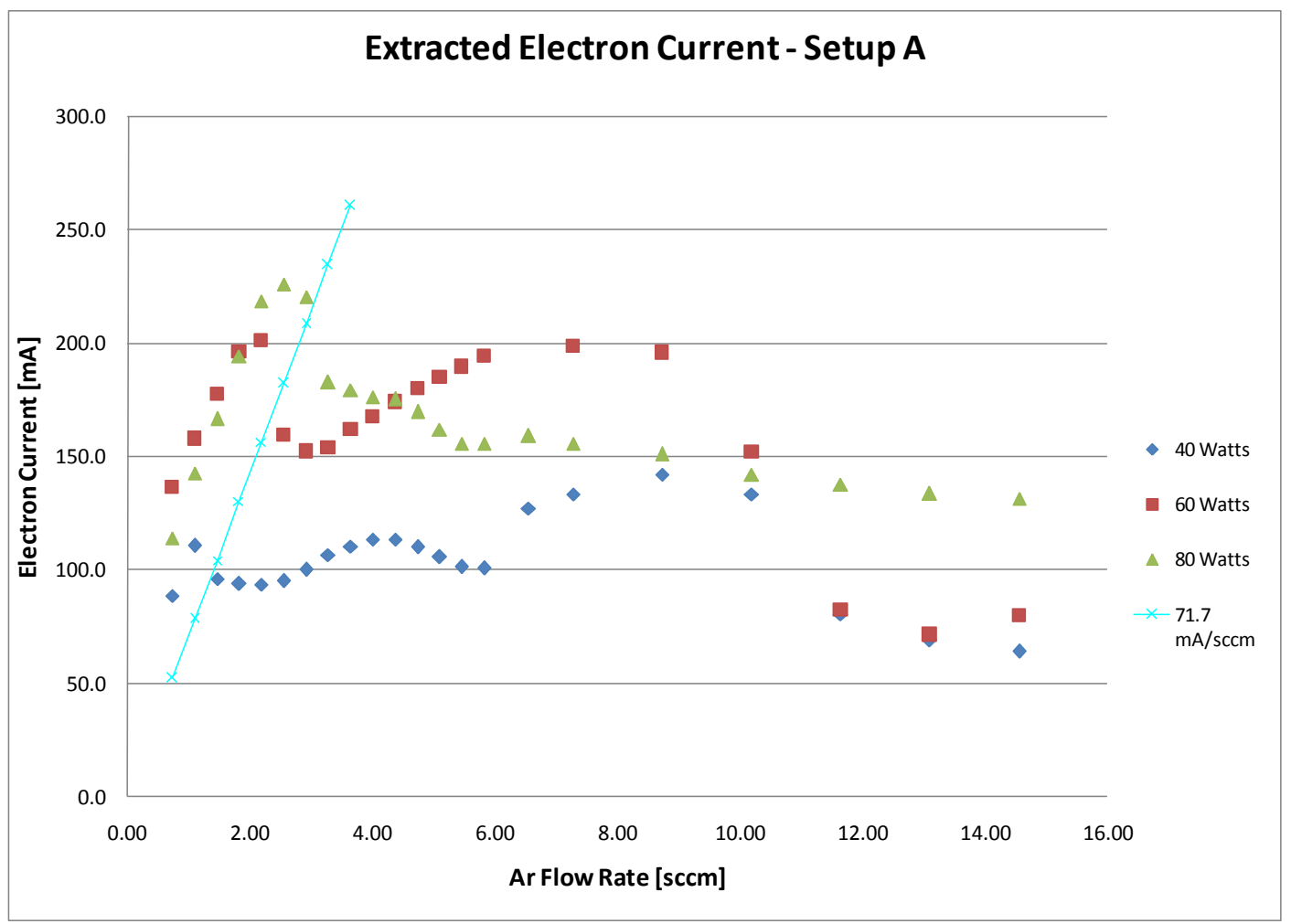

Figure 15. Extracted electron current as a function of power level, Setup A only.

\section{Current Extraction Tests - One Magnet per Slot}

Because of the fact that the strong magnetic field between the slots appeared to limit the amount of extractable current, the bottom magnet in each slot was removed. Two tests were run with the mica sleeve still in place, but with the electrode in two different locations, as shown in Fig. 16. Current measurements were taken in the same way as in the previous tests, with a $60 \mathrm{~V}$ electrode bias and a sweep of flow rates at 40,60 , and $80 \mathrm{~W}$ of absorbed power.

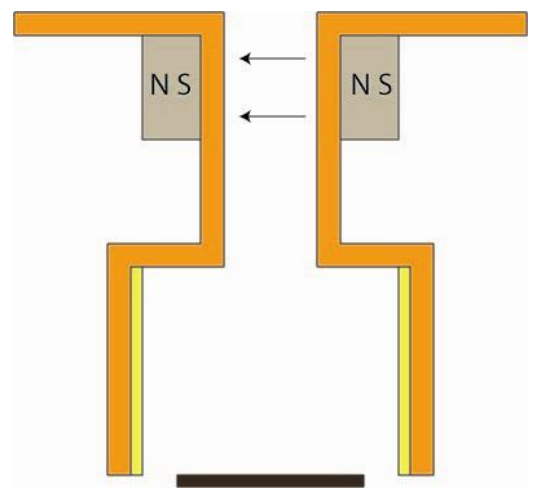

a) "Setup D"

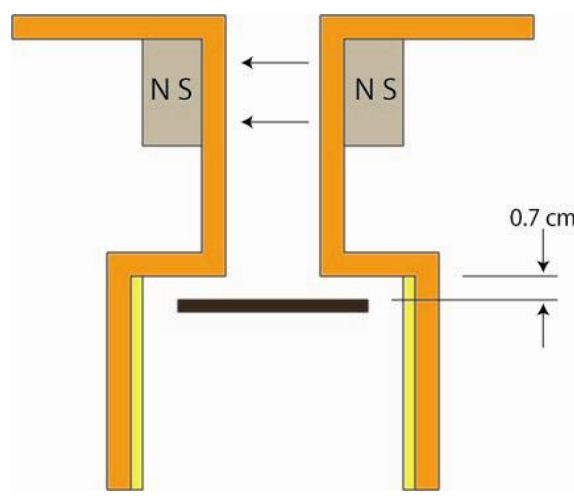

e) "Setup E"

Figure 16. Electrode configurations investigated during current extraction tests with 1 magnet per slot.

A comparison of the extracted electron current for Setups A through E are shown in Fig. 17, as a function of flow rate at $60 \mathrm{~W}$ of absorbed power. In setups $\mathrm{D}$ and $\mathrm{E}$, it there does not seem to be any ion recycling taking place at low flow rates, as opposed to Setup A. With the removal of the bottom magnets, the region where the magnetic 
field is strong enough for resonance heating was dramatically reduced in size. The neutralized ion residence time in the plasma production zones decreased as a result, and this reduced the chance that an ion could be recycled before being collected at the wall.

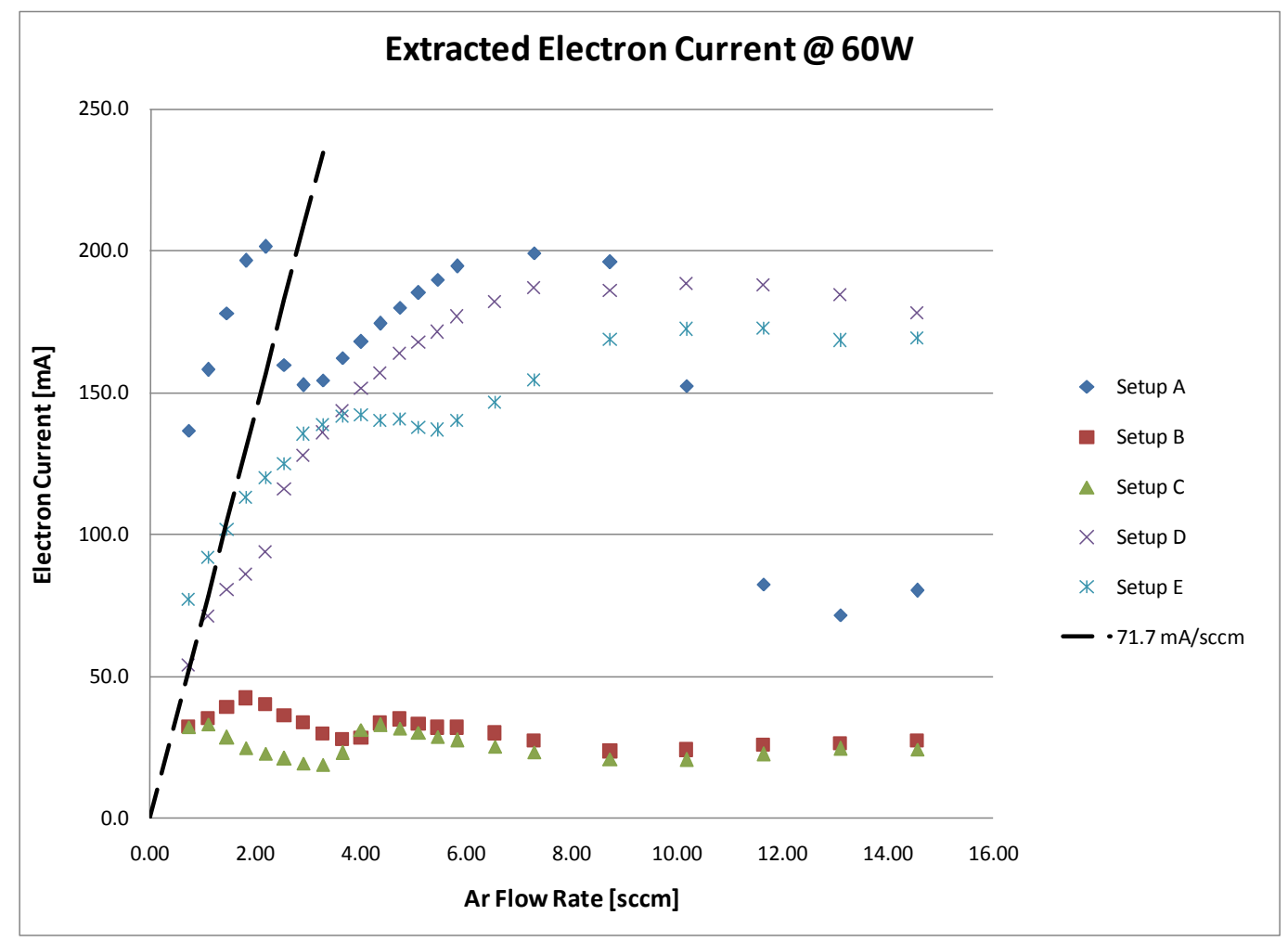

Figure 17. Extracted electron current as at 60 Watts, for Setups A through E.

The magnetic field strength at the electrode in Setup D was negligible, and it was 20 Gauss in Setup E. Since the electrode in Setup A had many more field lines terminating on it than in Setup E, it is expected that the extracted current in Setup E would be smaller than in Setup A. This is supported by the data at low flow rates, where electrons are essentially tied to the field lines. The difference between extracted current in Setup D and Setup E, however, is not easily explained. The plasma production region and wall loss area is the same in both setups, and there were no problems with microwave circuit matching in either setup. The reason that the extracted current is smaller when the electrode is closer to the discharge could be that the gas feed is situated below the electrode in Setup D. The electrode can act as a baffle, which restricts gas flow into the plasma production region, but unfortunately there are no pressure data from within the tube itself which can verify or refute this explanation.

Although the extracted current from in Setups D and E is slightly less than the current in Setup A at the higher flow points, the single magnet geometry appears to be the more attractive option. The electrons in the double magnet geometry need to diffuse across a much larger high B-field region than in the single magnet geometry. The large extracted current obtained in Setup A was entirely due to highly magnetized electrons which likely followed the magnetic field lines to the electrode surface. When the electrode was moved to the exit plane of the tube, the extracted current dropped by a factor of 5 to 10 at most flow rates. Since the discharge electrons are completely localized to the plasma production region in these setups, it may difficult to use them to seed an orifice discharge at the end of the tube. The single-magnet geometry does allow discharge electrons to escape the plasma production region. In effect, the bottom magnets were acting as a magnetic "shield" which isolated the plasma production region from the rest of the source. The extracted current in Setup D, where the electrode is at the exit plane of the tube, is actually slightly larger than the extracted current in Setup E at most flow rates. It is also comparable to the extracted current in Setup A over the range of flow rates from 3.2 to $8.8 \mathrm{sccm}$. 


\section{Langmuir Probe Traces - One Magnet per Slot}

The electrode in Setup E was replaced by two cylindrical Langmuir probes shown in Fig. 11. The probes were mounted to the tube, on axis and $0.7 \mathrm{~cm}$ from the bottom of the magnet slots, where the electrode was placed in previous tests. One probe was oriented along the axis of the tube, while the other probe was pointed parallel to the main component of the magnetic field. The source was run with one magnet in each slot, similar to Setup E, in order to determine the plasma characteristics at the electrode. Probe traces were recorded using the SmartProbe software at a range of flow rates and at absorbed power levels of 40,60, and 80 Watts. To approximate the actual electron density and temperature at the probes, average values of these quantities were calculated from the results of the axial and transverse probe traces. The mean electron density extracted from these traces is shown as a function of flow rate and power level in Fig. 18.

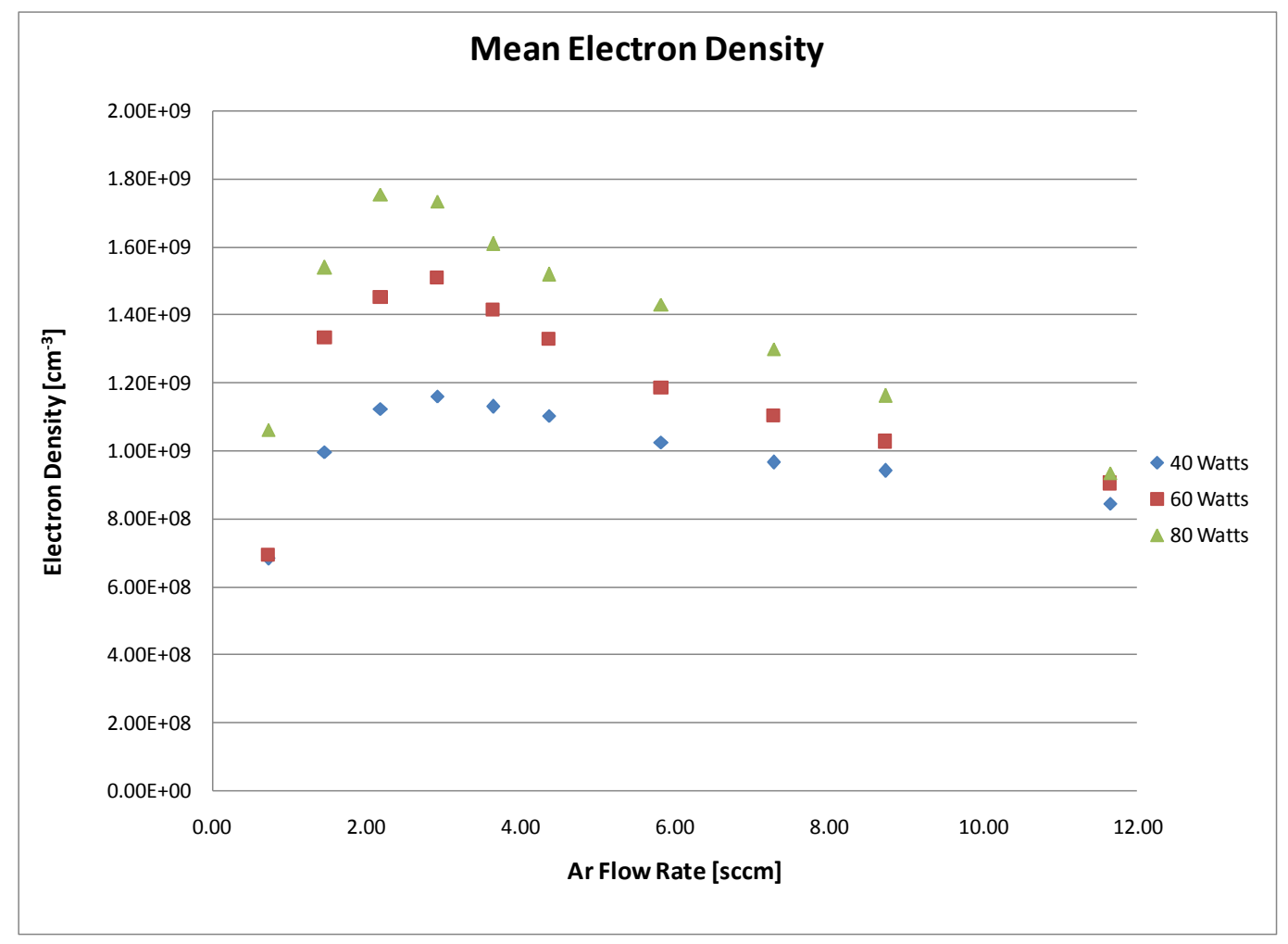

Figure 18. Electron density from Langmuir probe traces.

As expected, the electron density peaks as the flow rate increases, and then decreases as the flow rate is increased further. At low flow rates, the increase in flow rate feeds more neutrals into the source, which results in more ionization and a higher electron density. At flow rates higher than about $2.5 \mathrm{sccm}$, the density drops with flow rate because in this region, the ionization rate is decreasing with flow rate. As the flow rate goes up, the collision frequency increases as well, so each electron completes less cyclotron orbits between collisions. The ultimate energy of the ionizing electrons decreases with flow rate as a result of this. It is likely that above flow rates of 2.5 sccm, the ionization cross section is dropping faster than the neutral density is increasing, since the ionization cross section decreases rapidly as the electron energy decreases. This would naturally result in a lower ionization rate and therefore, a lower plasma density. The density increases with the absorbed power level over all flow rates, as more energy is deposited into the discharge.

For $2.45 \mathrm{GHz}$ microwaves, the cutoff plasma density from Eq. (5) is $7.44 \times 10^{10} \mathrm{~cm}^{-3}$, which is over an order of magnitude larger than the highest measured plasma density in Fig. 18. This is likely due to the electron heating mechanism in the source. When the magnets are placed in attraction across the slots, it is expected that upper hybrid heating is the dominant source of ionization. In upper hybrid heating, the fixed microwave frequency is equal to the upper hybrid frequency, given by Eq. (3).

In this respect, the plasma density will always be less than the microwave frequency equivalent of the plasma frequency; that is, the plasma density will always be less than cutoff. Consider the setup were upper hybrid heating is satisfied. An increase in the microwave power results in an increase in plasma density. Locally, upper hybrid 
heating is no longer satisfied by the propagating wave. Instead the wave is absorbed on the surface of the original heating zone where density and magnetic field strength are sufficiently reduced, in order to satisfy the hybrid heating conditions. In this respect, as density builds the heating surface moves outward. Here heating increases the density so that even at the reduced magnetic field, the condition is satisfied for heating. The input power is therefore spread out over a larger area leading to reduced microwave field strength per unit area. In the limit of the magnetic field goes to zero, the wave is simply reflected as an ordinary wave. In this respect, increases in power can increase the plasma production volume as well as the density up to cutoff, but not beyond. Therefore, there may be an upper limit on the plasma density, where the plasma is being produced, due to the upper hybrid heating mechanism between the magnet slots.

The probe traces showed little difference in electron temperature between the axial and transverse Langmuir probes, so in order to determine a general trend for the electron temperature, the two temperatures were averaged at each operating condition. The mean electron temperature from the probe traces is shown in Fig. 19, as a function of flow rate and power level. As expected, the temperature does not depend at all on the absorbed power level. When the power level increases at a fixed flow rate, a larger number of electrons have enough energy to ionize neutrals, but the mean collision time remains the same. This results in an increase in density. It is peculiar that the electron temperature is nearly independent of flow rate, since one should expect the mean energy of the electrons to decrease as the pressure increases, and therefore the collision rate, increases within the source. Further measurements are required to ascertain the origin of this behavior.

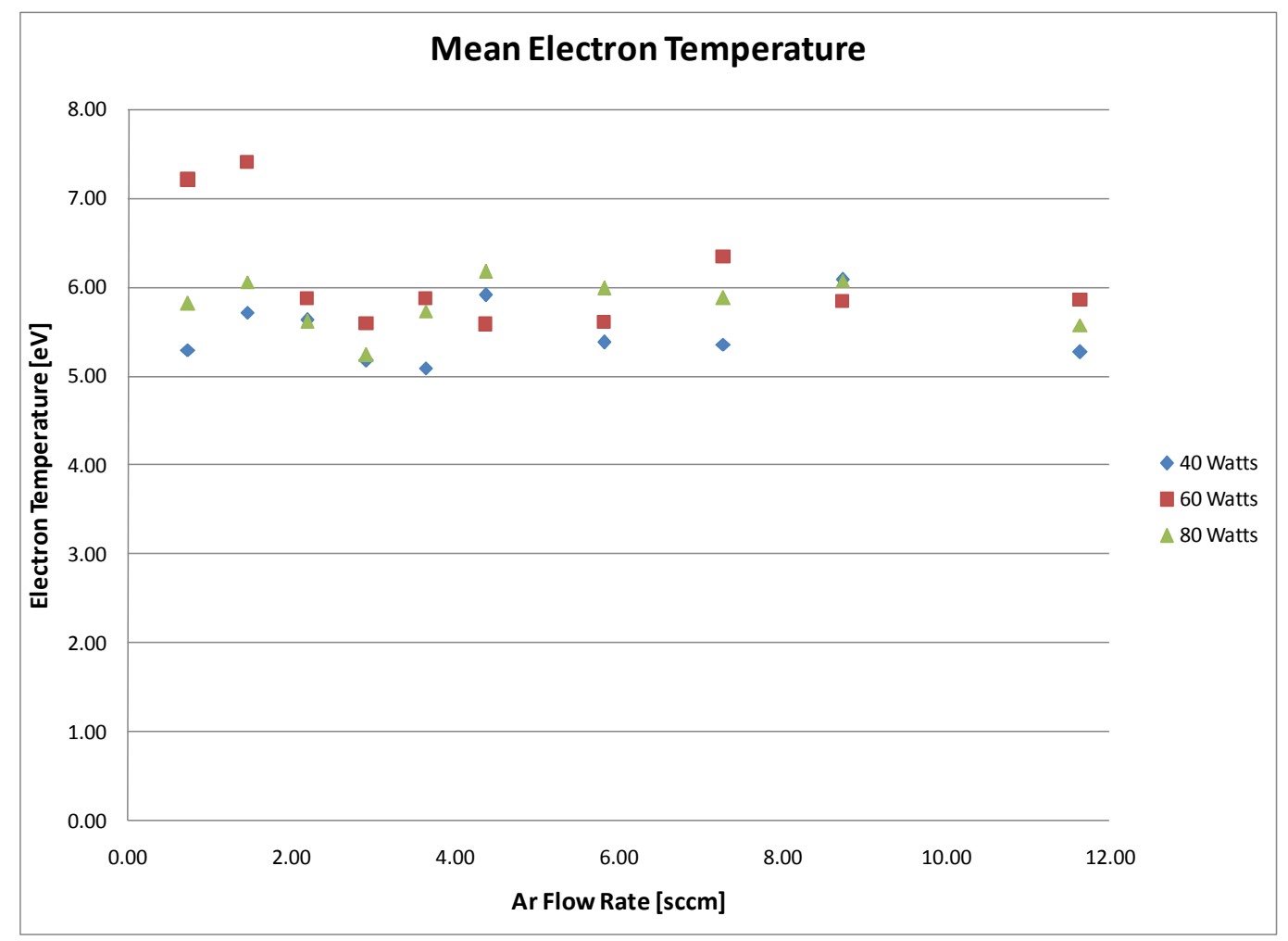

Figure 19. Mean electron temperature from Langmuir probe traces.

The electron densities and temperatures were used to estimate the amount of current that should be collected by the electrode biased at electron saturation voltages. This "theoretical" current was determined from,

$$
I_{t h}=A_{p} \times \frac{1}{4} n e \sqrt{\frac{8 k T_{e}}{\pi m}}
$$

The theoretical current closely follows the shape of the electron density curves in Fig. 18, and since the electron temperature appears to be independent of flow rate, this is to be expected. The probe data appear to indicate that the plasma is highly non-uniform because the measured and theoretical current values do not coincide at most flow rates. Another interesting point is that even at 80 Watts, the theoretical current value never reaches over $300 \mathrm{~mA}$. 
Since the theoretical current value depends strongly on number density, this implies that in order to improve upon the amount of extractable current, the plasma density at the electrode must be increased. All of the data does seem to suggest that the amount of extractable current is highly peaked at the range of flow rates from 2-4 sccm.

\section{Conclusion}

The waveguide plasma source described in this paper has not yet been optimized for electron current extraction, but it does show some promise as an electron source for an ion beam neutralizer. Most of the tests that have been carried out thus far include the variation of the permanent magnet geometry, which is used to set up ECR and upper hybrid heating zones within the source. Several magnet geometries have been tried, and it appears that the virtual cusps resulting from the magnets being placed in repulsion are responsible for large electron losses to the walls of the source. With the magnets in attraction, a more stable discharge is achieved, but the magnetic field tends to suppress the amount of extractable current. This geometry may also limit the number density of the discharge if the dominant ionization source is upper hybrid heating.

The initial characterization of the source has led to some insights about the source as it is, as well as some ideas about how to improve the source. It is clear that the plasma needs to be well-confined in order to have stable operation within the waveguide tube. However, any confining magnetic field oriented perpendicular to the axis of the tube will severely limit the amount of current that can be extracted at the exit plane of the tube. For this reason, the magnetic circuit needs to be modified in order to confine the plasma with a predominantly axial magnetic field, which should facilitate electron diffusion to the exit plane of the tube. In addition, an axial magnetic field of 875 Gauss will set up ECR zones, as opposed to upper hybrid zones, which will allow plasma densities up to and above the cutoff density to be achieved.

The discharge that has been studied thus far is only intended to act as a "seed plasma," which will supply electrons for an orifice discharge at the end of the source. The orifice and keeper electrode to be used with the seed plasma have yet to be designed, but the use of an orifice and keeper is expected to increase the amount of current that can be extracted from the source.

\section{References}

${ }^{1}$ Rayman, M. D., Varghese, P., Lehman, D.H., and Livesay, L. L., "Results from the Deep Space 1 Technology Validation Mission," Acta Astronautica, Vol. 47, Nos. 2-9, 2000, pp. 475-487

${ }^{2}$ Kuninaka, et al., "Powered Flight of HAYABUSA in Deep Space," Proc. $42^{\text {nd }}$ AIAA/ASME/SAE/ASEE Joint Propulsion Conference and Exhibit, 9-12 July, 2006, Sacramento, CA, AIAA Paper 2006-4318

${ }^{3}$ Koppel, C. R., Marchandise, F., and Prioul, M., "The SMART-1 Electric Propulsion Subsystem around the Moon: In Flight Experience," Proc. 41 ${ }^{\text {st }}$ AIAA/ASME/SAE/ASEE Joint Propulsion Conference and Exhibit, 10-13 July, 2005, Tucson, AZ, AIAA Paper 2005-3671

${ }^{4}$ Brophy, J. R., et al., "Development and Testing of the Dawn Ion Propulsion System," Proc. 42 ${ }^{\text {nd }}$ AIAA/ASME/SAE/ASEE Joint Propulsion Conference and Exhibit, 9-12 July, 2006, Sacramento, CA, AIAA Paper 2006-4319

${ }^{5}$ Kininaka, H. and Satori, S., "Development and Demonstration of a Cathodeless Electron Cyclotron Resonance Ion Thruster," Journal of Propulsion and Power, Vol. 14, No. 6, Nov-Dec. 1998, pp. 1022-1026

${ }^{6}$ Fusellier, C., et al., "A Microwave Plasma Cathode Electron Gun for Ion Beam Neutralization," Review of Scientific Instruments, Vol. 69, No. 2, Feb. 1998, pp. 1153-1155

${ }^{7}$ Korzec, D., Müller, A., and Engemann, J., "Microwave Plasma Source for High Current Ion Beam Neutralization," Review of Scientific Instruments, Vol. 71, No. 2, Feb. 2000, pp. 800-803

${ }^{8}$ Diamant, K., "Resonant Cavity Hollow Cathode," Proc. $41^{\text {st }}$ AIAA/ASME/SAE/ASEE Joint Propulsion Conference and Exhibit, 10-13 July, 2005, Tucson, AZ, AIAA Paper 2005-3662

${ }^{9}$ Foster, J. E., and Patterson, M. J., "Characterization of 40-Centimeter Microwave Electron Cyclotron Resonance Ion Source and Neutralizer," Journal of Propulsion and Power, Vol. 21, No. 5, Sept-Oct 2005, pp. 862-869

${ }^{10}$ Hidaka, Y., Foster, J. E., Getty, W. D., Gilgenbach, R. M., and Lau, Y. Y., "Performance and Analysis of an Electron Cyclotron Resonance Plasma Cathode," Journal of Vacuum Science and Technology A, Vol. 25, No. 4, July/Aug. 2007, pp. 781790

${ }^{11}$ Rao, Y., Zhang, Y., Wu, J., and Qi, L., "Design and Investigation of a Microwave Plasma Cathode," Review of Scientific Instruments, Vol. 65, No. 4, April 1994, pp. 1316-1318.

${ }^{12}$ Edgar, M. C., and Bilén, S. G., "Design and Testing of a High Power Electron Cyclotron Resonance Neutralizer," Proc. $43^{\text {rd }}$ AIAA/ASME/SAE/ASEE Joint Propulsion Conference and Exhibit,8-11 July, 2007, Cincinnati, OH, AIAA Paper 2007-5289

${ }^{13}$ Pozar, D.M., Microwave Engineering, John Wiley and Sons, 2005

${ }^{14}$ Lieberman, M.A., and Lichtenberg, A.J., Principles of Plasma Discharges and Materials Processing, John Wiley and Sons, 2005, pp. 87-129 